\title{
Transition metal-free photodecarboxylative amination of redox- active esters with diazirines
}

\author{
Vishala Maharaj, ${ }^{1}$ Preeti P. Chandrachud, ${ }^{2}$ Wen Che, ${ }^{2}$ Lukasz Wojtas, ${ }^{1}$ Justin M. Lopchuk ${ }^{1,2,3 *}$ \\ ${ }^{1}$ Department of Chemistry, University of South Florida, Tampa, FL 33620, USA \\ ${ }^{2}$ Drug Discovery Department, H. Lee Moffitt Cancer Center and Research Institute, 12902 Magnolia Drive, Tampa, FL \\ 33612, USA. \\ ${ }^{3}$ Department of Oncologic Sciences, College of Medicine, University of South Florida, Tampa, FL 33612, USA.
}

\begin{abstract}
Due to the prevalence of nitrogen-containing compounds in medicine, materials, and related fields, the search for general, mild methods for electrophilic amination remains an area of intense research. Diazirines have been recently demonstrated to serve as electrophilic amination reagents that afford diaziridines, versatile heterocycles that are readily transformed into amines, hydrazines, and a variety of nitrogen-containing heterocycles. Here we report the phosphine-mediated, photodecarboxylative amination of redox-active esters with diazirines. This method is transition metal-free, uses inexpensive photoactivators under mild conditions, and offers a significantly enhanced scope and yields for primary redox-active esters. Furthermore, the stability of diazirines to blue light $(456 \mathrm{~nm})$ is demonstrated, paving the way for further research into other photochemical amination methods with these unique heterocycles.
\end{abstract}

Nitrogen-containing compounds are ubiquitous in pharmaceuticals, agrochemicals, catalysts/ligands, materials, and more. ${ }^{1}$ As a result, the development of methods for $\mathrm{C}-\mathrm{N}$ bond formation continues unabated. Despite their popularity and obvious utility in a variety of areas, ${ }^{2}$ the use of redox-active esters (1, RAEs) for $\mathrm{C}-\mathrm{N}$ bond formation has been relatively underexplored. Of these few reports, all have used a transition metal/organophotoredox approach that was combined with copper catalysis (Figure 1A). The method developed by Peters and $\mathrm{Fu}$ recaptures the phthalimide after cleavage of the primary or secondary RAE to afford protected amines as the final products. ${ }^{3}$ Over the last several years, Hu has disclosed three methods that convert alkyl RAEs to aminated products. The first of these uses a ruthenium catalyst with an oxoacetic acidbased ligand and copper halide that yields anilines with primary or secondary RAEs. ${ }^{4}$ This was followed by an iridium-and copper-catalyzed method that uses an electron-deficient bisarylated imine with primary, secondary, and tertiary RAEs ${ }^{5}$ and, finally, an organophoto- and copper-catalytic method that can furnish either anilines or imines depending on the nitrogen source used. ${ }^{6}$ Recently we reported the use of diazirines and an iron- or nickel-catalyzed system that reacts with RAEs to afford diaziridines. ${ }^{7}$

While historically neglected by synthetic chemists, diaziridines 3 have now been demonstrated to be highly useful intermediates in the synthesis of amines, hydrazines, and numerous nitrogen-containing heterocycles, such as pyrazoles, pyrroles, triazoles, pyridazinones, and others (Figure 1B), ${ }^{7,8}$ Perfluorinated diazirines have also been shown to be effective in combination with fluorous phase synthesis. ${ }^{7}$ The main limitation in our previously reported work was low reactivity of primary RAEs with diazirine 4 (Figure 1C). This was partially alleviated by the use of a perfluorinated diazirine instead of $\mathbf{4}$,

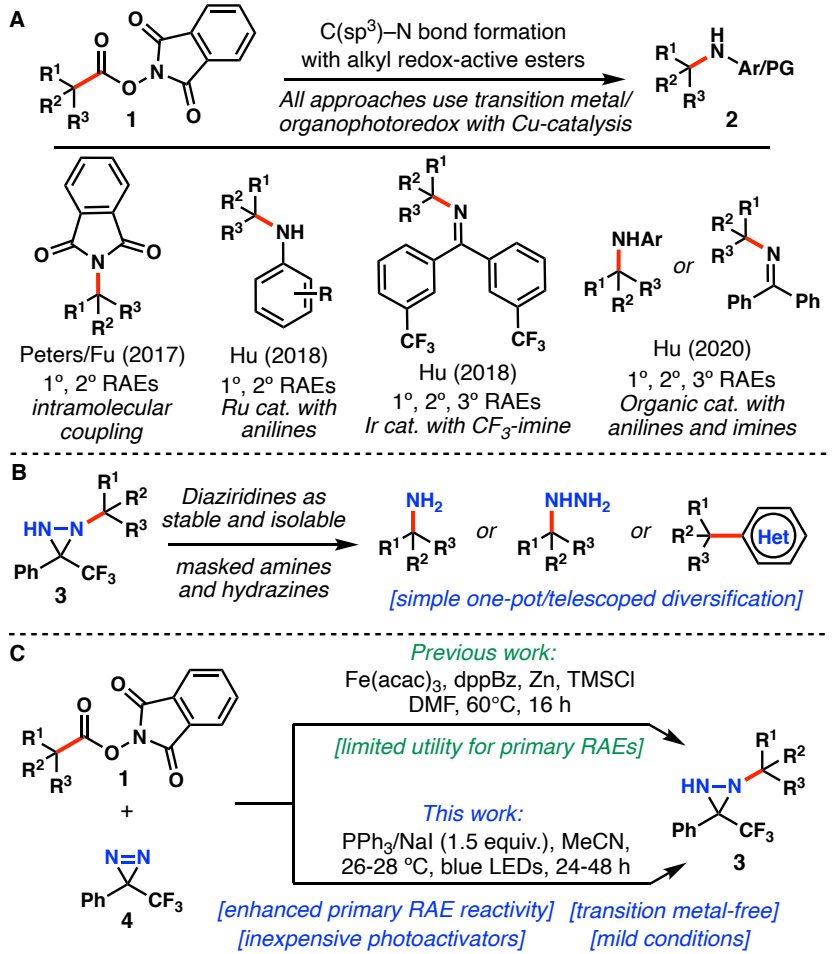

Figure 1. A. Previously reported methods for the amination of redox-active esters. B. Diazirines as masked amines and hydrazines. C. Comparison of the decarboxylative amination of redox-active esters with diazirines under iron-catalyzed conditions to photoredox conditions (this work). 
which showed sufficient reactivity to deliver the desired products in synthetically useful yields. However, diazirine $\mathbf{4}$ is not only commercially available, but also more economical and able to be prepared in fewer steps. Therefore, we elected to develop a complementary approach that allowed for the use of diazirine 4 in the decarboxylative amination of redox-active esters.

To overcome the limitation of the amination of primary RAEs with diazirine 4, we sought alternative activation modes for 1. In 2019, Shang and $\mathrm{Fu}$ reported the triphenylphosphine/sodium iodide-mediated photocatalytic decarboxylative alkylation of redox-active ester $\mathbf{5}$ with silyl enol ether $\mathbf{6}$ (Figure $2 \mathrm{~A}$ ). ${ }^{9}$ This approach was particularly appealing since it uses inexpensive, readily available photoactivators that form electron donor-acceptor (EDA) complexes in the presence of blue light, ${ }^{10}$ avoiding expensive transition metal catalysts or exotic organocatalysts. ${ }^{11}$ More recently this transformation has been achieved with a simple ammonium iodide catalyst in an amide solvent, which proceeds through an anion$\pi$ interaction in a solvent cage (Figure 2A). ${ }^{12}$

Initial attempts to directly translate the published catalytic conditions to the reaction of redox-ester 8 with diazirine 4 led to the desired diaziridine 9 in $19 \%$ yield (Figure 2B, entry 1, see SI for full details). Repeated attempts to optimize the catalytic reaction with respect to phosphine (entries 3 and 6), solvent, LED type/placement, and temperature were unsuccessful. Instead, using 1.5 equivalents of both triphenylphosphine and sodium iodide led to 9 in $76 \%$ yield. A screen of phosphines (entries 2, 4, 5, and 7-10) revealed several $(\mathbf{1 0}, \mathbf{1 2}, \mathbf{1 4})$ that worked in moderate to good yields $(52-70 \%)$, but none as economically as $\mathrm{PPh}_{3}$. Both acetone (entry 11) and DMA (entry 12) afforded the desired product with some diminishment in yield but could be considered in cases where substrates are poorly soluble in acetonitrile. The temperature of the reactions proved critical (entries $13-15$ ), with $26-28{ }^{\circ} \mathrm{C}$ providing optimal yields of diaziridine 9. Reactions below $18{ }^{\circ} \mathrm{C}$ were sluggish in addition to giving lower yields; in reactions above 38 ${ }^{\circ} \mathrm{C}$ only traces of product were observed. Finally, the use of 1.5 equivalents of tetrabutylammonium iodide (TBAI) furnished diaziridine 9 in 54\% yield and could represent an alternative set of conditions for this transformation.

Given that diazirines are typically photoactivated to form the corresponding carbenes, ${ }^{13}$ it was somewhat surprising to observe the near complete stability to blue LEDS over several days. Diazirine $4\left(\lambda_{\max }=353 \mathrm{~nm}\right)^{14}$ is stable to blue LEDs (456 nm, 2 lamps, each $3 \mathrm{~cm}$ from reaction vial) as a solution in cyclohexane for at least 48 hours. Small amounts of degradation are observed in acetonitrile at 16 hours, though most of diazirine 4 is still present at 48 hours (see SI for details). However, when exposed to a $500 \mathrm{~W}$ halogen lamp, diazirine 4 decomposes completely within six hours in either cyclohexane or acetonitrile ( 4 has been reported to have a half-life of 25 seconds with a $450 \mathrm{~W}$ mercury lamp placed 4 inches away). ${ }^{14}$ After 24 hours of ambient light exposure, a solution of diazirine 4 in acetonitrile shows small amounts of degradation. Alternatively, the exposure of $\mathbf{4}$ to ambient light as a neat compound begins to show degradation after 6 days (which demonstrates 4's ease of handling as a reagent, see SI for details). In order to probe the stability of $\mathbf{4}$ to blue LEDs further, competition experiments were run with the optimized reaction conditions where RAE 8, diazirine 4 and cyclohexane (17, 1 equiv.

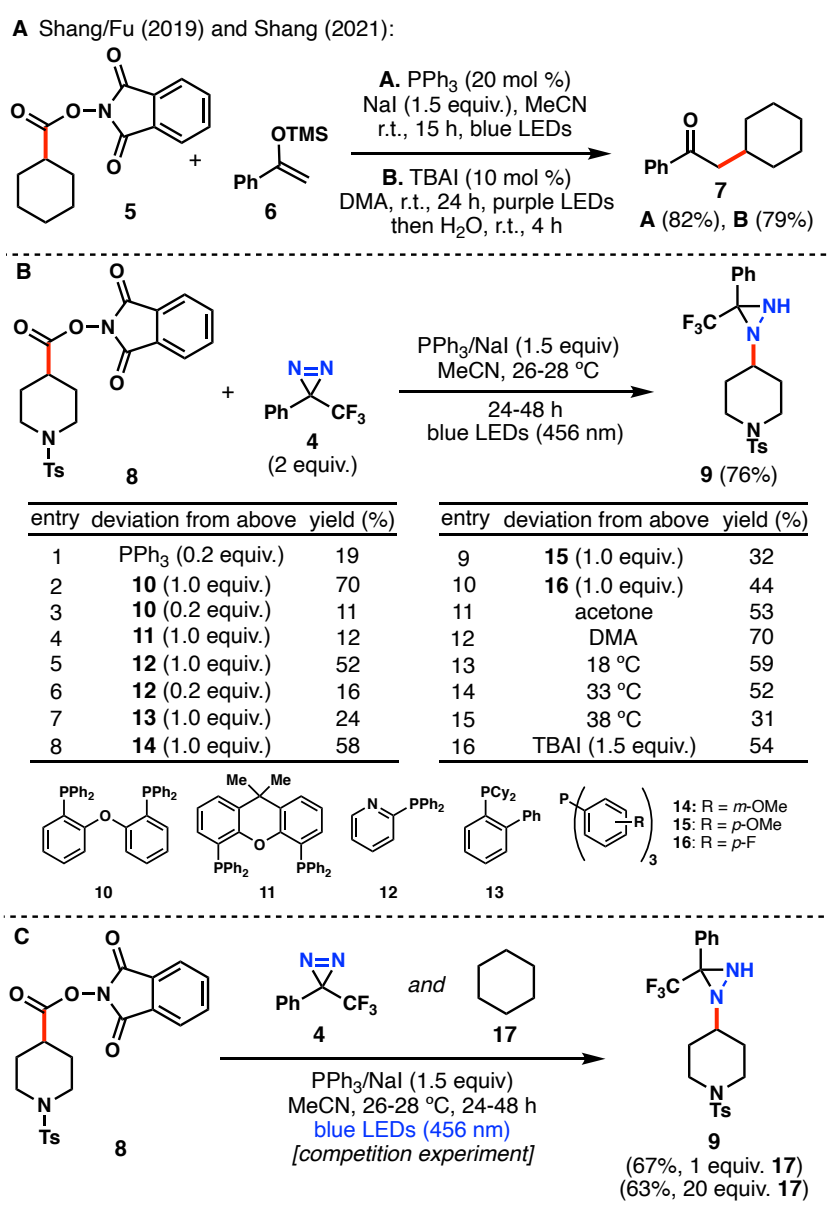

Figure 2. A. Phosphine/iodide-mediated decarboxylative amination of redox-active esters with silyl enol ethers. B. Optimization of the photodecarboxylative amination of redoxactive esters with diazirines. C. Competition experiment of diazirine $\mathbf{4}$ and cyclohexane (17) with blue LEDs.

and 20 equiv.) were exposed to blue LEDs for $40 \mathrm{~h}$ (Figure $2 \mathrm{C}$ ). The target diaziridine 9 was isolated in $67 \%$ yield (1 equiv. 17) and $63 \%$ yield (20 equiv. 17) after full consumption of RAE 8. In contrast, when the reaction was set up with a 500 W halogen lamp, only traces of diaziridine 9 were detected with nearly all of diazirine 4 decomposed within 90 minutes.

With optimized conditions in hand, the scope was evaluated, starting with primary redox-active esters (Figure 3). Gratifyingly, treatment of the redox-active ester derived from 4phenylbutanoic acid with diazirine $\mathbf{4}$ afforded diaziridine $\mathbf{1 9}$ in $60 \%$ yield, significantly improved over the previous ironcatalyzed conditions (variable $9-31 \%$ yield). ${ }^{7}$ Several more direct comparisons with primary redox-active esters were made including: alkyl chloride 20 (71\% vs. 36\% with $\mathrm{Fe}$ ), alkyne 21 (57\% vs. 17\% with Fe), ester 22 (74\% vs. 30\% with $\mathrm{Fe}$ ), and dehydrocholic acid derivative $\mathbf{4 0}$ (84\% vs. traces with $\mathrm{Fe})$. Numerous natural products and pharmaceuticals bearing primary carboxylic acids were amenable to the amination process including linoleic acid (24, 53\%), mycophenolic acid (29, $50 \%)$, glutamic acid (30-33, 45-50\%), 2,4-D (35, 56\%), fenbufen $(36,50 \%)$, gabapentin $(39,44 \%)$, dehydrocholic acid (40, 84\%), and atorvastatin $(41,45 \%)$. The treatment of the redox-active ester of 3-phenylbutanoic acid under the diaziridine-forming conditions led to "amphetamine diaziridine" 37 

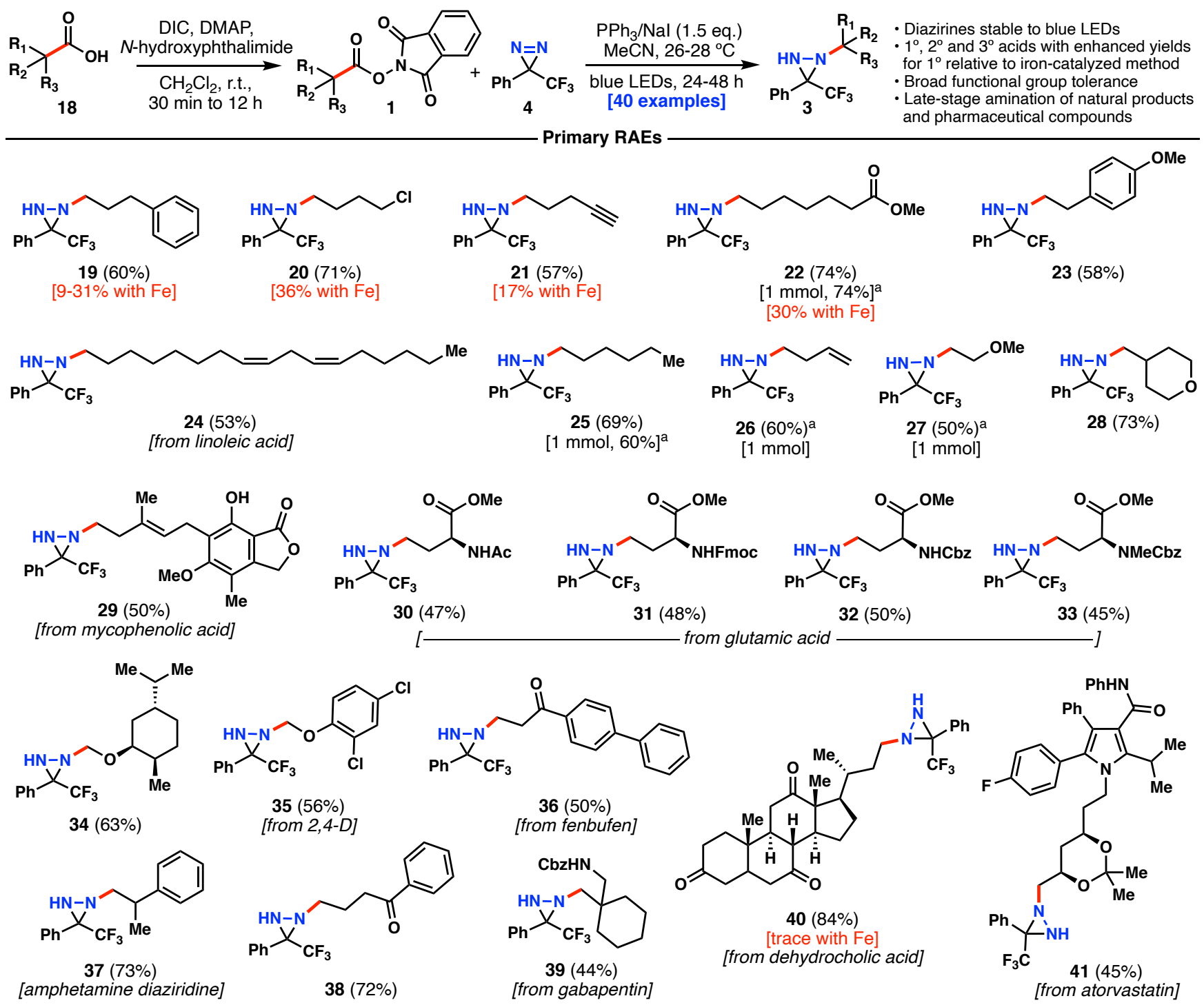

Figure 3. Scope of the photodecarboxylative amination of primary redox-active esters with diazirines. General reaction conditions: RAE (1 equiv.), diazirine (2 equiv.), $\mathrm{PPh}_{3}$ (1.5 equiv.), $\mathrm{NaI}\left(1.5\right.$ equiv.), $\mathrm{MeCN}(0.1 \mathrm{M})$, blue LEDs (2 lamps, $3 \mathrm{~cm}$ from reaction vial), 26-28 ${ }^{\circ} \mathrm{C}$, 24-48 h. 3 equiv. of diazirine were used.

in $73 \%$ yield. While the mono-aza amphetamine class of compounds are well studied, ${ }^{15}$ this approach also provides rapid access to the hydrazinyl versions. These di-aza derivatives have been reported to possess a wide variety of activities including antihypertensive, ${ }^{16}$ analeptic, ${ }^{17}$ antidepressive, ${ }^{18}$ and antibacterial, ${ }^{19}$ while acting as $\mathrm{MAO}^{17}$ and $\mathrm{D}$ amino acid oxidase inhibitors, ${ }^{20}$ and have even been explored as amphetamine prodrugs. ${ }^{21}$

Glutamic acids 30-33 are notable due to the straightforward and economical access to the corresponding hydrazine derivatives without epimerization. The hydrazines, particularly methylated versions related to $\mathbf{3 3}$, have previously been used in the synthesis of vancomycin aglycon analogs. ${ }^{22}$ Their literature preparation comes from expensive homoserine derivatives (compared to inexpensive, readily available glutamic acid) in a fairly low-yielding process over 5-6 steps. ${ }^{22}$

As anticipated from the success with the primary RAEs, both secondary and tertiary RAEs worked well, furnishing the desired diaziridines in moderate to high yields. In addi- tion to commonly used building blocks for medicinal chemistry (e.g. 9, 47-50), menthyl formic acid (45), gemfibrozil (52), and oleanolic acid (55) were successfully aminated. Interestingly, there were several examples where the photoredox method outperformed the iron-catalyzed approach: tetrahydrofuran derivative $\mathbf{4 2}$ (92\% vs. 38\% with Fe), ketone 49 ( $85 \%$ vs. $42 \%$ with Fe), and difluoro 47 (88\% vs. $53 \%$ with Fe). Fluorous phase diaziridines $\mathbf{5 6}$ and $\mathbf{5 7}$ were also prepared in good yields, which allows the photoredox amination to be coupled with a high throughput library synthesis for the rapid preparation and purification of diverse nitrogencontaining compounds. ${ }^{7,23}$

Overall, the structural diversity of the primary, secondary, and tertiary RAEs was vast with a broad functional group tolerance including alkyl halides (20), alkynes (21), esters $(22,30-33,56)$, ethers $(23,27,29,34,35,52)$, olefins $(24$, 26, 29, 55), heterocycles, $(9,28,41,42,46,51,54,57)$, hydroxy/phenols $(\mathbf{2 9}, \mathbf{4 8}, \mathbf{5 5})$, lactones $(\mathbf{2 9})$, ketones $(\mathbf{3 6}, \mathbf{3 8}$, 40, 49), acetals (41), sulfones (46), and silyl ethers (50). Compatible amine protecting groups include Ac (30), Fmoc 

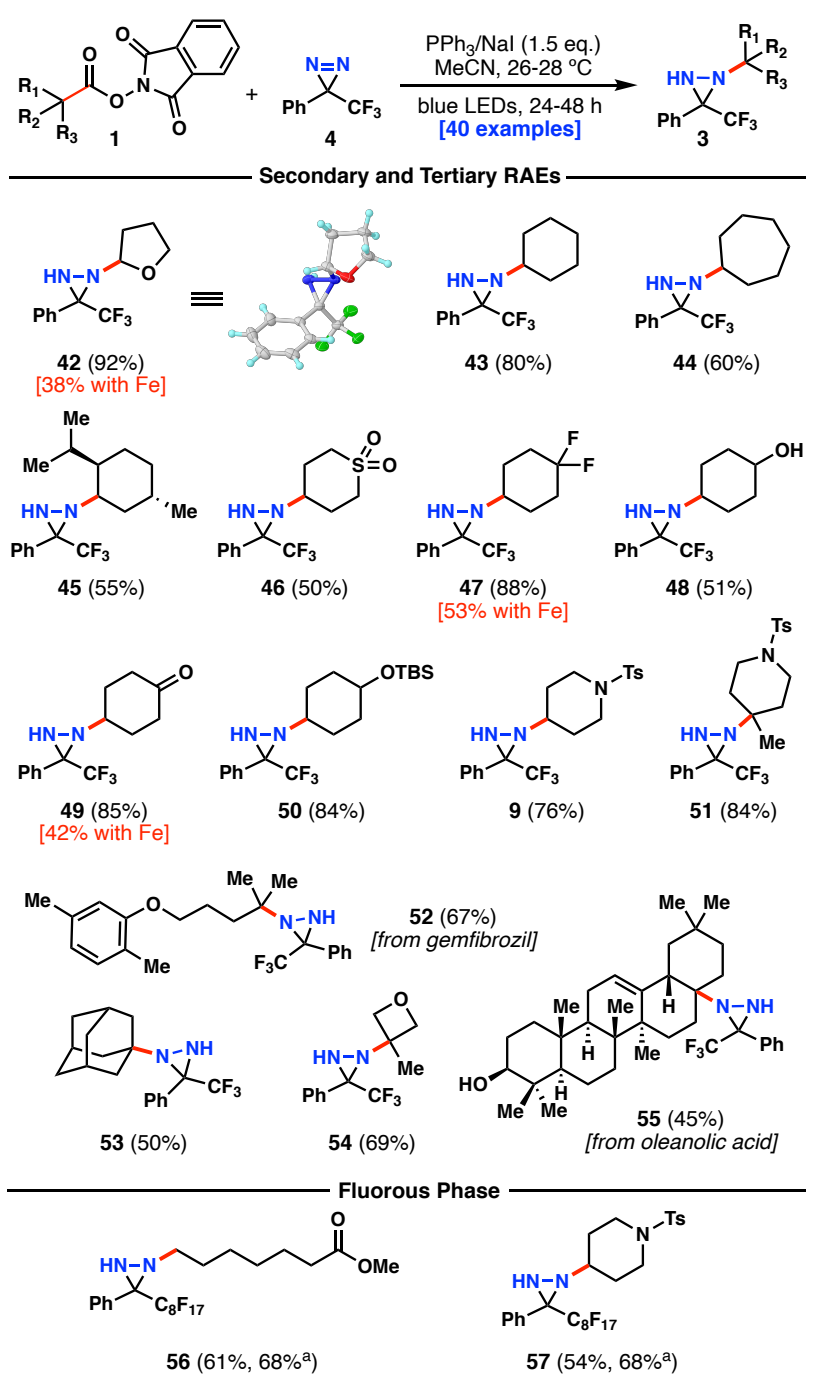

Figure 4 Scope of the photodecarboxylative amination of secondary and tertiary redox-active esters with diazirines. General reaction conditions: RAE (1 equiv.), diazirine (2 equiv.), $\mathrm{PPh}_{3}$ (1.5 equiv.), $\mathrm{NaI}$ ( 1.5 equiv.), $\mathrm{MeCN}(0.1 \mathrm{M})$, blue LEDs (2 lamps, $3 \mathrm{~cm}$ from reaction vial), $26-28^{\circ} \mathrm{C}, 24-48 \mathrm{~h} .{ }^{\mathrm{a}} 3$ equiv. of diazirine were used.

(31), $\mathrm{Cbz}(\mathbf{3 2}, \mathbf{3 3}, \mathbf{3 9})$, and Ts $(\mathbf{9}, \mathbf{5 1}, \mathbf{5 7})$. In addition to the tertiary RAEs (51-55), other sterically hindered examples include diaziridines 39 and $\mathbf{4 5}$. The reactions were scaled to $1 \mathrm{mmol}$ with similar yields to the rest of the scope $(\mathbf{2 2}, \mathbf{2 5}$ 27).

In conclusion, we have reported a transition metal-free photodecarboxylative amination of redox-active esters with diazirines that proceeds under mild conditions with inexpensive, readily available triphenylphosphine and sodium iodide photoactivators. ${ }^{24}$ The scope and yields of primary RAEs (24 examples) are significantly improved over our previously reported method. Some functional group tolerance, especially with ketones, has also been improved. While the reasons for the observed differences in reactivity between the ironcatalyzed and photoredox methods are not fully understood, experiments are ongoing and the results will be reported in due course. As has been previously demonstrated, the diaziridines obtained from this reaction are easily converted to amines, hydrazines, and nitrogen-containing heterocycles. The demonstration of the exceptional compatibility of blue
LEDs and diazirines that allows for the formation of amination products is expected to facilitate the exploration of related new methods; efforts toward this end are ongoing in our laboratory.

\section{ASSOCIATED CONTENT}

\section{Supporting Information}

The Supporting Information is available free of charge on the ACS Publications website.

General information, experimental details, stability studies, graphical procedures, and analytical data $\left({ }^{1} \mathrm{H},{ }^{13} \mathrm{C},{ }^{19} \mathrm{~F}\right.$ NMR, $\mathrm{MS}$ ) for all new compounds (PDF)

X-ray crystallographic data are available free of charge from the Cambridge Crystallographic Database Centre (2107759 (S55), 2107760 (42) (CIF)).

\section{AUTHOR INFORMATION}

\section{Corresponding Author}

* Justin M. Lopchuk

Email: justin.lopchuk@moffitt.org.

Notes

The authors declare no competing interests.

\section{ACKNOWLEDGMENT}

We thank Dr. Harshani Lawrence for assistance with NMR experiments, Chuan Shan for assistance with X-ray crystallography, and Dr. Zachary Shultz for assistance with data analysis. We thank Prof. Rui Shang for helpful discussions relating to the chemistry described in reference 9 . We gratefully acknowledge the Donors of the American Chemical Society Petroleum Research Fund (59537-DNI1) and the National Science Foundation (CHE-1903144) for support of this research. This work has also been supported in part by the Chemical Biology Core Facility at the H. Lee Moffitt Cancer Center \& Research Institute, an NCI designated Comprehensive Cancer Center (P30-CA076292).

\section{REFERENCES}

1. Lawrence, S. A. Editor. Amines: Synthesis, Properties and Applications, Cambridge University Press: Cambridge, 2004.

2. (a) Muraka, S. $N$-(Acyloxy)phthalimides as Redox-active Esters in Cross-coupling Reactions. Adv. Synth. Catal. 2018, 360, 1735-1753; (b) Niu, P.; Li, J.; Zhang, Y.; Huo, C. One-electron Reduction of Redox-active Esters to Generate Carbon-centered Radicals. Eur. J. Org. Chem. 2020, 5801-5814.

3. Zhao, W.; Wurz, R. P.; Peters, J. C.; Fu, G. C. Photoinduced, Copper-catalyzed Decarboxylative C-N Coupling to Generate Protected Amines: An Alternative to the Curtius Rearrangement. J. Am. Chem. Soc. 2017, 139, 12153-12156.

4. Mao, R. Z.; Frey, A.; Balon, J.; Hu, X. Decarboxylative $\mathrm{C}\left(\mathrm{sp}^{3}\right)-N$ Cross-coupling via Synergetic Photoredox and Copper Catalysis. Nat. Catal. 2018, 1, 120-126.

5. Mao, R. Z.; Balon, J.; Hu, X. Cross-coupling of Alkyl Redoxactive Esters with Benzophenone Imines: Tandem Photoredox and Copper Catalysis. Angew. Chem. Int. Ed. 2018, 57, 9501-9504.

6. Barzanó, G.; Mao, R.; Garreau, M.; Waser, J.; Hu, X.; Tandem Photoredox and Copper-catalyzed Decarboxylative $\mathrm{C}\left(\mathrm{sp}^{3}\right)-\mathrm{N}$ Coupling of Anilines and Imines using an Organic Photocatalyst. Org. Lett. 2020, 22, 5412-5416.

7. Chandrachud, P. P.; Wojtas, L.; Lopchuk, J. M. Decarboxylative Amination: Diazirines as Single and Double Nitrogen Transfer Reagents. J. Am. Chem. Soc. 2020, 142, 21743-21750. 
8. Schneider, Y.; Prevost, J.; Gobin, M.; Legault, C. Y. Diazirines as Potent Electrophilic Nitrogen Sources: Application to the Synthesis of Pyrazoles. Org. Lett. 2014, 16, 596-599.

9. Fu, M.-C.; Shang, R.; Zhao, B.; Wang, B.; Fu, Y. Photocatalytic Decarboxylative Alkylations Mediated by Triphenylphosphine and Sodium iodide. Science 2019, 363, 1429-1434.

10. (a) Crisenza, G. E. M.; Mazzarella, D.; Melchiorre, P. Synthetic Methods Driven by the Photoactivity of Electron DonorAcceptor Complexes. J. Am. Chem. Soc. 2020, 142, 5461-5476; (b) Zheng, L.; Cai, L.; Tao, K.; Xie, Z.; Lai, Y.-L.; Buo, W. Progress in Photoinduced Radical Reactions using Electron Donor-acceptor Complexes. Asian J. Org. Chem. 2021, 10, 711-748.

11. (a) Prier, C. K.; Rankic, D. A.; MacMillian, D. W. C. Visible Light Photoredox Catalysis with Transition Metal Complexes: Applications in Organic Synthesis. Chem. Rev. 2013, 113, 5322-5363; (b) Romero, N. A.; Nicewicz, D. A. Organic Photoredox Catalysis. Chem. Rev. 2016, 116, 10075-10166.

12. (a). Wang, G.-Z.; Fu, M.-C.; Zhao, B.; Shang, R. Photocatalytic Decarboxylative Alkylations of $\mathrm{C}\left(\mathrm{sp}^{3}\right)-\mathrm{H}$ and $\mathrm{C}\left(\mathrm{sp}^{2}\right)-\mathrm{H}$ Bonds Enabled by Ammonium Iodide in Amide Solvent. Sci. China Chem. 2021, 64, 439-444; (b) Liu, C.; Shen, N.; Shang, R. Photocatlytic Decarboxylative Alkylation of Silyl Enol Ether and Enamide with $\mathrm{N}$-(acyloxy)phthalimide using Ammonium Iodide. Org. Chem. Front. 2021, 8, 4166-4170.

13. Das, J. Aliphatic Diazirines as Photoaffinity Probes for Proteins: Recent Developments. Chem. Rev. 2011, 111, 4405-4417.

14. Brunner, J.; Senn, H.; Richards, F. M. 3-Trifluoromethyl-3phenyldiazirine. A New Carbene Generating Group for Photolabeling Reagents. J. Biol. Chem. 1980, 255, 3313- 3318.

15. Heal, D. J.; Smith, S. L.; Gosden, J.; Nutt, D. J. Amphetamine, Past and Present - a Pharmacological and Clinical Perspective. J. Psychopharmacol. 2013, 27, 479-496.

16. Bream, J. B.; Picard, C. W.; White, T. G. Aralkylaminoguanidines and Related Compounds. J. Med. Chem. 1970, 13, 10511057.

17. Biel, J. H.; Drukker, A. E.; Mitchell, T. F.; Sprengeler, E. P.; Nuhfer, P. A.; Conway, A. C.; Horita, A. Central Stimulants. Chemistry and Structure-activity Relationships of Aralkyl Hydrazines. $J$. Am. Chem. Soc. 1959, 81, 2805-2813.

18. Elslager, E. F.; Weinstein, E. A.; Worth, D. F. Synthesis and Biological Properties of Aminoalkylhydrazines. A Unique Nitrogen-nitrogen Scission of 1-(2-diethylaminoethyl)-2-(1-phenyl-2propyl)hydrazine. J. Med. Chem. 1964, 7, 493-500.

19. Westland, R. D.; Holmes, J. L.; Green, B.; Dice, J. R. NSubstituted Derivatives of 2-aminoethanethiol and 2hydrazinoethanethiol. J. Med. Chem. 1968, 11, 824-829.

20. Tsukamoto, T.; Slusher, B. S.; Ferraris, D. V.; Rojas, C.; Hin, N., Duvall, B. Inhibitors of D-amino Acid Oxidase. PCT Intl. Appl. WO 2014/025993 A1. Feb. 13, 2014.

21. Whomsley, R.; Allan, C. E.; Luker, T. J. Amphetamine Prodrugs. PCT Intl. Appl. WO 2014/002039 A1. Jan. 3, 2014.

22. Crane, C. M.; Boger, D. L. Synthesis and Evaluation of Vancomycin Aglycon Analogues that Bear Modifications in the Nterminal D-leucyl Amino Acid. J. Med. Chem. 2009, 52, 14711476.

23. Zhang, W.; Curran, D. P. Synthetic Applications of Fluorous Solid-phase Extraction (F-SPE). Tetrahedron 2006, 62, 1183711865.

24. During the preparation of this manuscript a related diazirinebased decarboxylative amination of redox-active esters appeared. See: Shu, X.; Xu, R.; Liao, S. Photocatalytic Divergent Decarboxylative Amination: A Metal-free Access to Aliphatic Amines and Hydrazines. Sci. China Chem. 2021. doi:10.1007/s11426-021-10484. 